\title{
Reconstituted yogurt from yogurt cultured milk powder mix has better overall characteristics than reconstituted yogurt from commercial yogurt powder
}

\author{
Lijie Song and Kayanush J. Aryana ${ }^{1}$ \\ School of Animal Sciences, Louisiana State University Agricultural Center, Baton Rouge 70803
}

\begin{abstract}
For manufacture of commercial yogurt powder, yogurt has to go through a drying process, which substantially lowers the yogurt culture counts, so the potential health benefits of the yogurt culture bacteria are reduced. Also, upon reconstitution, commercial yogurt powder does not taste like yogurt and has an off-flavor. The objective was to study the microbial, physicochemical, and sensory characteristics of reconstituted yogurt from yogurt cultured milk powder (YCMP) mix and reconstituted yogurt from commercial yogurt powder (CYP). The CYP reconstituted yogurt was the control and YCMP mix reconstituted yogurt was the treatment. Microbial and physicochemical characteristics of the CYP reconstituted yogurt and YCMP mix reconstituted yogurt were analyzed daily for the first week and then weekly for a period of $8 \mathrm{wk}$. Sensory consumer testing of CYP reconstituted yogurt and YCMP mix reconstituted yogurt was conducted with 100 consumers. At 56 d, YCMP mix reconstituted yogurt had $5 \log \mathrm{cfu} / \mathrm{mL}$ higher counts of Streptococcus thermophilus than the control (CYP reconstituted yogurt). Also, Lactobacillus bulgaricus counts of YCMP mix reconstituted yogurt were $6.55 \log \mathrm{cfu} / \mathrm{mL}$ at $28 \mathrm{~d}$ and were $5.35 \log \mathrm{cfu} / \mathrm{mL}$ at $56 \mathrm{~d}$, whereas the CYP reconstituted yogurt from $28 \mathrm{~d}$ onwards had a count of $<10 \mathrm{cfu} / \mathrm{mL}$. The YCMP mix reconstituted yogurt also had significantly higher apparent viscosity and sensory scores for appearance, color, aroma, taste, thickness, overall liking, consumer acceptability, and purchase intent than CYP reconstituted yogurt. Overall, YCMP mix reconstituted yogurt had more desirable characteristics than CYP reconstituted yogurt.

Key words: yogurt, powder, reconstituted
\end{abstract}

\section{INTRODUCTION}

According to the Code of Federal Regulations (21 CFR 131.200), yogurt is the food produced by culturing

Received March 28, 2014.

Accepted July 8, 2014

${ }^{1}$ Corresponding author: karyana@agcenter.lsu.edu cream, milk, partially skim milk, or skim milk, used alone or in combination, with lactic acid-producing bacteria Lactobacillus delbrueckii ssp. bulgaricus and Streptococcus thermophilus. Yogurt has a titratable acidity (TA) of not less than $0.9 \%$, expressed as lactic acid (21 CFR 131.200). The yogurt market has grown considerably since 2003. Yogurt sales and consumption have increased in the last 5 yr (IDFA 2009, 2010, 2011, 2012, 2013). Generally, the recommended total number of active yogurt culture bacteria at the time of consumption should be a minimum of $10^{7} \mathrm{cfu} / \mathrm{g}$ (Chandan, 1999). To confer health benefits, probiotic bacteria should be viable at the time of consumption at a recommended concentration of 6 to 8 log cfu/g (Ross et al., 2005; Vasiljevic and Shah, 2008).

Many potential health and nutritional benefits from lactic acid bacteria exist. These include improved digestion of lactose and control of intestinal infections, some types of cancer, and serum cholesterol levels (Gilliland, 1990). Some L. delbrueckii ssp. bulgaricus strains have immunological effects (Ebina et al., 1995; Kitazawa et al., 1998; Kitazawa et al., 2003). Certain strains of $S$. thermophilus produce bacteriocins. Some studies have characterized several bacteriocins (e.g., thermophilin 110 and thermophilin 1277) produced by $S$. thermophilus that are active against Pediococcus acidilactici, Clostridium butylicum, Clostridium sporogenes, Clostridium botulinum, Bacillus cereus, and Listeria monocytogenes (Gilbreth and Somkuti, 2005; Kabuki et al., 2009). To improve the health benefits, the recent trend is to add Lactobacillus acidophilus to yogurt (Ashraf and Shah, 2011). Lactobacillus acidophilus may prevent diarrhea in children and adults, and it is especially effective in treating rotavirus in children (Lee et al., 2001; Allen et al., 2009). Other health benefits associated with $L$. acidophilus include anticarcinogenic properties, reduction in blood pressure and serum cholesterol concentration, and increased resistance to infectious diseases (Ashraf and Shah, 2011).

Generally, yogurt is dried by spray drying, microwave vacuum drying, or freeze-drying. Each drying method has its benefits and drawbacks. Survival of yogurt bacteria is affected by the outlet temperature in the spray drying process. According to Bielecka and Majkowska 
(2000), the best survival at an outlet temperature range of 70 to $75^{\circ} \mathrm{C}$ was 13.7 to $15.8 \%$ for L. delbrueckii ssp. bulgaricus and 51.6 to $54.7 \%$ for $S$. thermophilus. At temperatures below $60^{\circ} \mathrm{C}$, wet powder was obtained, whereas at above $90^{\circ} \mathrm{C}$, the powder was not acceptable due to browning (Kim and Bhowmik, 1990). In addition, it has been reported that most of the aroma compounds and rheological characteristics of yogurt are lost during the spray-drying process (Kumar and Mishra, 2004). The approximate survival of culture bacteria was $50 \%$ in microwave vacuum-dried yogurt below $45^{\circ} \mathrm{C}$ (Kim et al., 1997). Freeze-dried yogurt has the best flavor and is the more authentic product compared with those obtained using other conventional drying methods (Rybka and Kailasapathy, 1997). The survival of lactic acid bacteria in freeze-dried yogurt was only 50 to $60 \%$ (Radaeva et al., 1975). Freeze-drying is the most expensive form of drying and generally not commercially feasible for manufacturing large quantities of yogurt powder.

Yogurt powder can be used in a wide variety of food applications, including instant yogurt and replacement of fresh yogurt for beverage and dip. It can also be used in snacks, confections, bakery items and breakfast cereals, ice cream bars, and yogurt-coated nuts. Upon reconstitution, yogurt powder does not taste like yogurt; it is off-flavored. However, yogurt powder provides longer and more stable shelf life than that of regular yogurt. Moreover, the reduced weight and bulk water of this dehydrated product decreases packaging, handling, and transportation costs. This product is very convenient for consumers to use, as it can be stored at ambient temperature for a long shelf life. Internationally, consumers do not always have access to purchasing natural yogurt in supermarkets. A powdered version can also be shipped to natural calamity areas or for food aid to less-fortunate countries. Other than providing nutrition, a food product also providing convenience in storage and consumption would be an added advantage. For a reconstituted yogurt, consumers would just need to add the powder to water and stir to mix well. The yogurt and yogurt drink market has benefited greatly as consumers have paid greater attention to healthy eating. In the food industry, it is important to produce a high-quality food product with a low cost. Upon reconstitution, having a better quality product than is currently available is desirable and would be beneficial to both the food industry and consumers. Further study on the parameters of this kind of reconstituted yogurt is also valuable. Industry manufactures and sells milk powder but preparation of reconstituted milk is an application at the consumer/ household end. This is also true for other industries, such as the vegetable juice powder and fruit juice powder industries. This same concept is being applied here to yogurt cultured milk powder (YCMP) mix, which can be a future industry endeavor, and reconstituted yogurt can be the application at the consumer end for convenience in storage and consumption.

The purpose of this study was to determine whether reconstituted YCMP mix would have higher culture bacterial counts and better physicochemical and sensory characteristics than reconstituted commercial yogurt powder (CYP) currently available. The specific objectives of this study were to (1) enumerate $S$. thermophilus, L. delbrueckii ssp. bulgaricus, Escherichia coli/coliforms, and yeasts and molds of reconstituted CYP and reconstituted YCMP mix up to $8 \mathrm{wk}$; (2) elucidate the influence on the physicochemical characteristics ( $\mathrm{pH}, \mathrm{TA}$, color, and apparent viscosity) of reconstituted CYP and YCMP mix up to 8 wk; and (3) study the sensory characteristics of CYP, YCMP mix, and YCMP mix with L. acidophilus (YCMPA) upon reconstitution and determine the consumer acceptability of the product.

\section{MATERIALS AND METHODS}

\section{Reconstituted Yogurt Manufacture}

Reconstituted yogurt formulations are shown in Table 1. The CYP was whey and nonfat milk mixed together, cultured, and spray dried to obtain the nonfat yogurt powder (DairiConcepts LP, Springfield, MO).

Commercial yogurt powder reconstituted yogurt was manufactured with nonfat yogurt powder (DairiConcepts LP), water, and blueberry puree (Sensient Tech-

Table 1. Reconstituted commercial yogurt powder (CYP) and reconstituted yogurt-cultured milk powder mix (YCMP) formulations

\begin{tabular}{lcc}
\hline & \multicolumn{2}{c}{ Formulation } \\
\cline { 2 - 3 } & CYP, & YCMP \\
Ingredient & $\mathrm{g}$ & mix, g \\
\hline Commercial yogurt powder & 948.4 & 0 \\
Nonfat dry milk & 0 & 810 \\
Water & 2,700 & 2,700 \\
Blueberry puree & 720 & 720 \\
Citric acid $^{2}$ & 0 & 23.4 \\
Pectin $^{3}$ & 0 & 72 \\
Yogurt starter culture $^{3}$ & 0 & 43 \\
\hline
\end{tabular}

${ }^{1}$ Sensient Technologies Corp. (Milwaukee, WI).

${ }^{2}$ Anhydrous, coarse granular 14 to 30 mesh (Sungai Budi Group, Jakarta Selatan, Indonesia).

${ }^{3}$ Low-methoxyl pectin (Gum Technology Inc., Tucson, AZ).

${ }^{4} \mathrm{~A}$ blend at a 1:1 ratio of Lactobacillus delbrueckii ssp. bulgaricus and Streptococcus thermophilus (FD-DVS YC-380; Chr. Hansen Inc., Milwaukee, WI). 
nologies Corp., Milwaukee, WI). Water was preheated to $40^{\circ} \mathrm{C}$ and nonfat yogurt powder was added to the $40^{\circ} \mathrm{C}$ water and stirred, which was followed by addition of blueberry puree and subsequent stirring to make CYP reconstituted yogurt, which was then cooled to $4^{\circ} \mathrm{C}$ until further analysis.

Yogurt cultured milk powder mix reconstituted yogurt was manufactured with instant, low-heat, nonfat dry milk; freeze-dried yogurt starter culture (a blend at a 1:1 ratio of $L$. delbrueckii ssp. bulgaricus and $S$. thermophilus; FD-DVS YC-380; Chr. Hansen Inc., Milwaukee, WI); pectin (low-methoxyl pectin; Gum Technology Inc., Tucson, AZ); citric acid (anhydrous, coarse granular 14-30 mesh; Sungai Budi Group, Jakarta Selatan, Indonesia); water; and blueberry puree (Sensient Technologies Corp.). After preheating the water to $40^{\circ} \mathrm{C}$, all dry ingredients were added to the $40^{\circ} \mathrm{C}$ water and stirred, which was followed by blueberry puree addition and subsequent stirring to make YCMP mix reconstituted yogurt, which was then cooled to $4^{\circ} \mathrm{C}$ until further analysis.

Total solids content in both CYP reconstituted yogurt and YCMP mix reconstituted yogurt were kept constant at $26 \%$ (wt/wt). Product manufacture was replicated 3 times.

Yogurt cultured milk powder mix with L. acidophilus reconstituted yogurt, for the sensory evaluation study, was manufactured by adding $1 \%$ (wt/wt) freeze-dried L. acidophilus (LYO 100 DCU-S, HOWARU; Chr. Hansen Inc.) to the YCMP mix. All dry ingredients were added to the $40^{\circ} \mathrm{C}$ water and stirred, which was followed by blueberry puree addition and subsequent stirring to make YCMPA mix reconstituted yogurt, which was then cooled to $4^{\circ} \mathrm{C}$.

\section{Experimental Design}

This study consisted of CYP reconstituted yogurt as the control and YCMP mix reconstituted yogurt as the treatment. For the first and second objectives, microbial and physicochemical characteristics of the reconstituted yogurts were analyzed daily for the first week and then weekly for a period of 8 wk $(1,2,3,4,5,6,7,14$, $21,28,35,42,49$, and $56 \mathrm{~d}$ ) after reconstitution. Three replications of each treatment were conducted. For the third objective, consumer tests of sensory characteristics of reconstituted yogurts from YCMP mix, YCMPA mix, and CYP were conducted with 100 panelists. The experimental design for sensory evaluation was a randomized block design with panelists as blocks. The experimental design for microbial counts, $\mathrm{pH}$, TA, and apparent viscosity was a randomized block design with repeated measures in time with replications as blocks.

\section{Preparation of Media}

Peptone water $(0.1 \%)$ was prepared by dissolving 1 $\mathrm{g}$ of peptone medium (Bacto Peptone, Difco; Becton, Dickinson and Co., Sparks, MD) in $1 \mathrm{~L}$ of distilled water, and then autoclaving in $99-\mathrm{mL}$ portions at $121^{\circ} \mathrm{C}$ for $15 \mathrm{~min}$. Streptococcus thermophilus agar and lactobacilli de Man, Rogosa, and Sharpe (MRS) agar with $\mathrm{pH}$ adjusted to 5.2 were prepared according to Dave and Shah (1996).

\section{Growth of S. thermophilus and L. delbrueckii ssp. bulgaricus}

Growth of S. thermophilus and L. delbrueckii ssp. bulgaricus was determined by pour plating. One gram of yogurt samples was serially diluted with $99 \mathrm{~mL}$ of sterilized $0.1 \%$ peptone water (Difco Laboratories Inc., Detroit, MI). One milliliter of diluted samples was pipetted into Petri dishes and then pour plated. Lactobacilli MRS agar adjusted to $\mathrm{pH} 5.2$ was prepared for L. delbrueckii ssp. bulgaricus and S. thermophilus agar for $S$. thermophilus. For $S$. thermophilus, pour plates were incubated aerobically at $37^{\circ} \mathrm{C}$ for $24 \mathrm{~h}$ and for $L$. delbrueckii ssp. bulgaricus, pour plates were incubated anaerobically at $43^{\circ} \mathrm{C}$ for $72 \mathrm{~h}$ before counting colonies (Dave and Shah, 1996; Tharmaraj and Shah, 2003).

\section{Escherichia coli/Coliform and Yeast and Mold Counts}

Escherichia coli/coliform and yeast and mold counts were performed after reconstitution of CYP and YCMP by using $E$. coli/coliform Petrifilm (3M, St. Paul, MN) containing violet red bile agar for $E$. coli/coliform, and yeast and mold Petrifilm (3M) for yeasts and molds. The procedure was performed by weighing $11 \mathrm{~g}$ of CYP and YCMP samples and diluting into $99 \mathrm{~mL}$ of sterilized $0.1 \%$ peptone water (Difco Laboratories Inc.) separately. Dilutions of $10^{-1}$ and $10^{-2}$ were prepared and $1 \mathrm{~mL}$ of the dilutions was plated in duplicate on previously labeled Petrifilm and incubated at $32^{\circ} \mathrm{C}$ for $24 \mathrm{~h}$ for E. coli/coliform and $25^{\circ} \mathrm{C}$ for $120 \mathrm{~h}$ for yeasts and molds. The colonies were counted after the incubation.

\section{pH}

The $\mathrm{pH}$ was measured after reconstitution of CYP and YCMP mix by using an Oysters Series $\mathrm{pH}$ meter (Extech Instruments Corp., Waltham, MA). The $\mathrm{pH}$ meter was calibrated using commercial pH 4.00 and 7.00 buffers (Fisher Scientific, Fair Lawn, NJ) and the 
temperature setting of the $\mathrm{pH}$ meter was adjusted to the temperature of sample $\left(8 \pm 2^{\circ} \mathrm{C}\right)$ before reading.

\section{$T A$}

The TA was measured after reconstitution of CYP and YCMP mix without any blueberry puree by weighing $9 \mathrm{~g}$ of yogurt, adding 5 drops of phenolphthalein as an indicator solution, and titrating with $0.1 \mathrm{~N} \mathrm{NaOH}$ to a faint pink end point.

\section{Apparent Viscosity}

The apparent viscosity was measured after reconstitution of CYP and YCMP mix using a viscometer (Brookfield model DV-II and Helipath Stand; Brookfield Engineering Laboratories Inc., Stoughton, MA). According to measurement recommendations, to obtain a torque between 10 and 90\%, 2 different spindles were used. For CYP, an RV1 spindle was used at a speed of $50 \mathrm{rpm}$ and for YCMP an RV4 spindle was used at a speed of $5 \mathrm{rpm}$. The RV spindle was inserted in the center of the sample at a constant depth of $2 \mathrm{~cm}$ from the top level of the sample. The Helipath was set in downward motion to cut circular layers at increasing depths of the sample. Dimensions of containers that held samples were as follows: top diameter $=7.5 \mathrm{~cm}$, bottom diameter $=6 \mathrm{~cm}$, and height $=9 \mathrm{~cm}$. The apparent viscosity was determined at $8 \pm 2^{\circ} \mathrm{C}$ and was continuous over the $33 \mathrm{~s}$ that were required to collect 100 data points acquired by the computer using Wingather 32 software (Brookfield Engineering Laboratories Inc.).

\section{Sensory Evaluation}

The sensory evaluation was exempted from continued oversight by the Louisiana State University Institutional Review Board through exemption number HE13-12. The sensory evaluation was conducted with 100 random participants including students and faculty at Louisiana State University. Three treatments: CYP, YCMP mix, and YCMPA mix, upon reconstitution, were packed into three $60-\mathrm{g}$ plastic cups and provided to participants in random order, using identical cups coded with 3-digit random numbers.

Participants were instructed not to talk to others during the evaluation and instructed to clean their palate between each sample with the purified water and unsalted saline crackers. The participants were given a sample of approximately $30 \mathrm{~g}$ per treatment per replication and were asked to evaluate it. The evaluation form consisted of a 9-point rating scale, ranging from 1 $=$ dislike extremely to $9=$ like extremely, to evaluate appearance, color, aroma, taste, thickness, powderiness, overall liking, and yes/no questions for acceptability and purchase intent of the products.

\section{Statistical Analysis}

The results for microbial and physicochemical characteristics were analyzed using PROC GLM of SAS (9.3; SAS Institute Inc., Cary, NC). Differences of least squares means were used to determine significant differences at $P<0.05$ for main effects (treatment and day) and interaction effect (treatment $\times$ day). Significant differences $(P<0.05)$ among the main effects were analyzed using the Tukey adjustment. The results for sensory evaluation were subjected to PROC ANOVA of SAS. Data are presented as mean \pm standard deviation of the mean.

\section{RESULTS AND DISCUSSION}

\section{Streptococcus thermophilus}

The counts of $S$. thermophilus in CYP and YCMP mix reconstituted yogurts during 8 wk of storage are shown in Figure 1. The treatment $\times$ day interaction effect was not significant $(P=0.077)$, whereas the treatment effect $(P<0.0001)$ and day effect $(P<0.0001)$ were significant.

Logarithmic mean counts of S. thermophilus in YCMP mix reconstituted yogurt were more than 3 times higher than logarithmic mean counts of $S$. thermophilus in CYP reconstituted yogurt (Figure 1). Because the CYP was manufactured by the spray-drying process, this significant difference may be explained by low survival rates during spray drying of the cultures and low stability during storage (Peighambardoust et al., 2011). The increase in the lag phase before the onset of growth means that spray-dried starter cultures cannot be used for inoculation in dairy fermentations directly (Boza et al., 2004).

During the storage period of $8 \mathrm{wk}$, the counts of $S$. thermophilus observed at $\mathrm{d} 1,2$, and 5 were significantly $(P<0.05)$ higher than the counts observed at d 28, 35, 42,49 , and 56. Michael et al. (2010) also reported that the counts of $S$. thermophilus declined from 9 to $8 \mathrm{log}$ $\mathrm{cfu} / \mathrm{mL}$ in yogurt during a storage period of $50 \mathrm{~d}$.

\section{Lactobacillus delbrueckii ssp. bulgaricus}

The counts of $L$. delbrueckii ssp. bulgaricus in CYP and YCMP reconstituted yogurts during the $8 \mathrm{wk}$ of storage are shown in Figure 2. The treatment $\times$ day interaction effect was significant $(P=0.0060)$. 


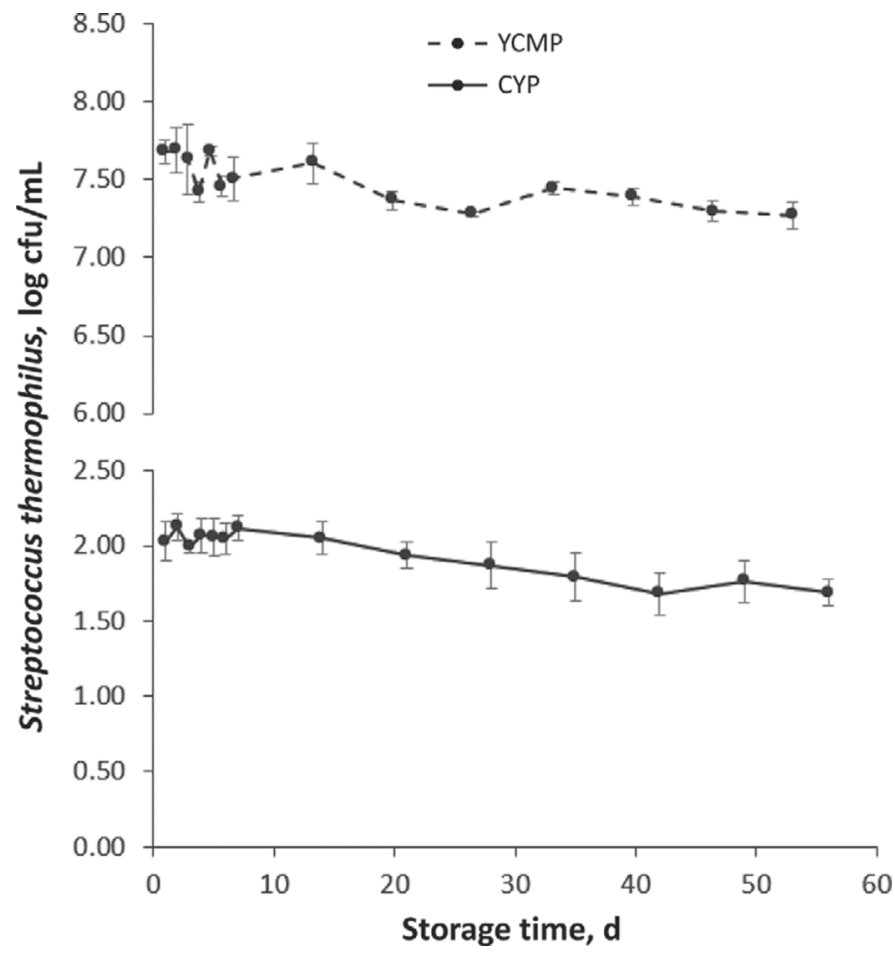

Figure 1. Logarithmic mean counts \pm SD of Streptococcus thermophilus for reconstituted yogurts from commercial yogurt powder (CYP) and yogurt cultured milk powder (YCMP) mix during $56 \mathrm{~d}$ of storage at $4 \pm 1^{\circ} \mathrm{C}$.

The YCMP mix reconstituted yogurt L. delbrueckii ssp. bulgaricus counts were significantly higher than the CYP reconstituted yogurt over $8 \mathrm{wk}$ of storage (Figure $2)$. In the spray-drying process, the number of $L$. delbrueckii ssp. bulgaricus decreased with increased outlet or inlet air temperature and atomizing air pressure (Kim and Bhowmik, 1990). Bielecka and Majkowska (2000) reported that the best survival was 13.7 to $15.8 \%$ for L. delbrueckii ssp. bulgaricus and 51.6 to $54.7 \%$ for $S$. thermophilus at an outlet temperature range of 70 to $75^{\circ} \mathrm{C}$. Therefore, it is likely that the commercial yogurt powder contained a low concentration of $L$. delbrueckii ssp. bulgaricus and S. thermophilus.

During the 8-wk storage period, the counts of $L$. delbrueckii ssp. bulgaricus in CYP reconstituted yogurt observed during the first $2 \mathrm{wk}(\mathrm{d} 1,2,3,4,5,6,7$, and 14) were significantly higher than the counts observed during wk 3. The viable counts in CYP reconstituted yogurt decreased to $<10 \mathrm{cfu} / \mathrm{mL}$ from d 28 onwards (Figure 2). The counts of L. delbrueckii ssp. bulgaricus in YCMP mix reconstituted yogurt observed during the first $2 \mathrm{wk}(\mathrm{d} 1,2,3,4,5,6,7$, and 14) were significantly higher than the counts observed during the last $6 \mathrm{wk}(\mathrm{d}$ $21,28,35,42,49$, and 56). Furthermore, the counts observed at d 21, 28, and 35 were significantly $(P<0.05)$ higher than counts observed at d 42, 49, and 56 (Figure 2 ). The higher counts of $L$. delbrueckii ssp. bulgaricus in CYP reconstituted yogurt were obtained in the first $3 \mathrm{wk}$ in the range of 1.86 to $0.67 \mathrm{log} \mathrm{cfu} / \mathrm{mL}$. Similarly, the higher counts of $L$. delbrueckii ssp. bulgaricus in YCMP mix reconstituted yogurt were obtained in the first 2 wk in the range of 7.52 to $7.14 \log \mathrm{cfu} / \mathrm{mL}$. The viable counts decreased to the lowest at $56 \mathrm{~d}$ (Figure 2). Michael et al. (2010) also reported that the growth of L. delbrueckii ssp. bulgaricus declined from 8 to $3 \log$ $\mathrm{cfu} / \mathrm{mL}$ in yogurt during a storage period of $50 \mathrm{~d}$.

\section{Escherichia coli/Coliforms, Yeasts, and Molds}

No E. coli/coliform bacteria were observed in either CYP reconstituted yogurt or YCMP reconstituted yogurt during the 8-wk storage period. Also, there were no detectable $E$. coli/coliform counts within the ingredients of CYP reconstituted yogurt and YCMP reconstituted yogurt. Therefore, it appears that no food safety issues would exist related to E. coli/coliforms in these reconstituted yogurt products. Growth of yeast and mold in CYP and YCMP reconstituted yogurts

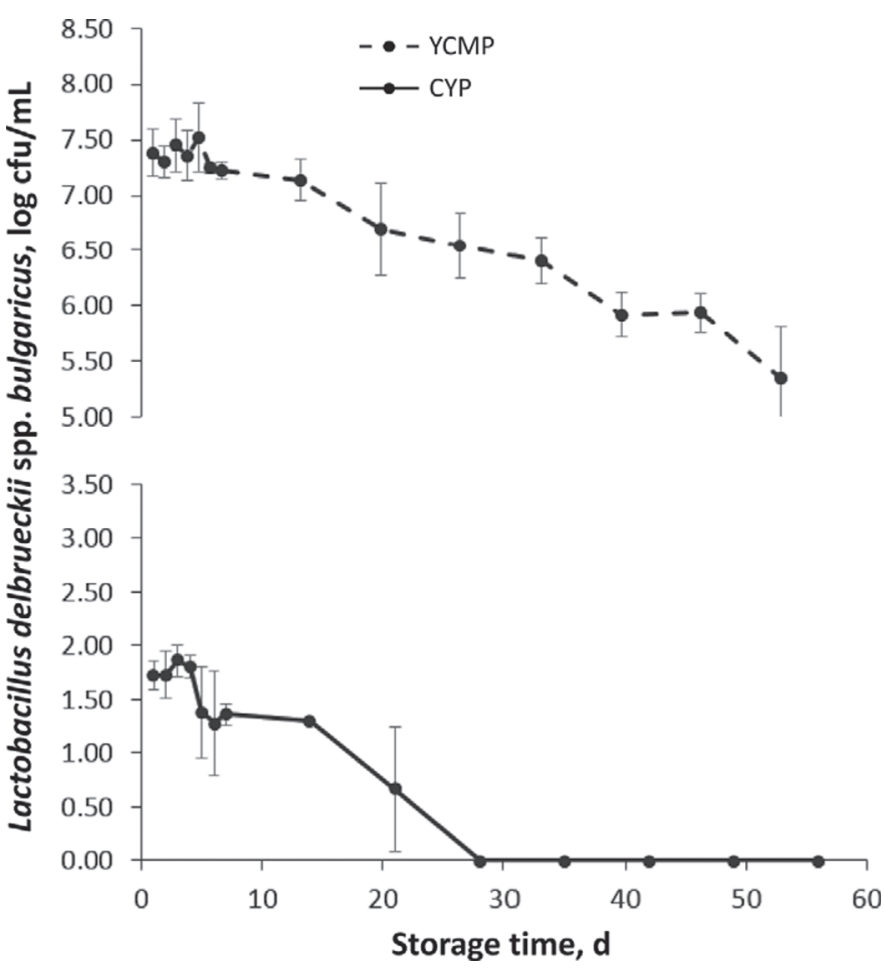

Figure 2. Logarithmic mean counts \pm SD of Lactobacillus delbrueckii ssp. bulgaricus for reconstituted yogurts from commercial yogurt powder (CYP) and yogurt cultured milk powder (YCMP) mix during $56 \mathrm{~d}$ of storage at $4 \pm 1^{\circ} \mathrm{C}$. 


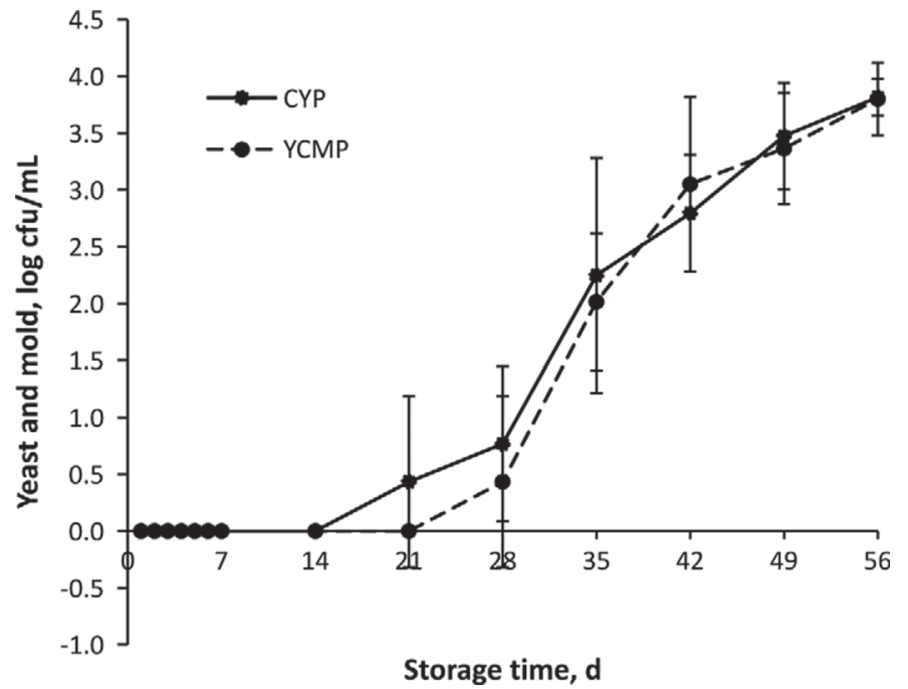

Figure 3. Logarithmic mean counts \pm SD of yeast and mold for reconstituted yogurts from commercial yogurt powder (CYP) and yogurt cultured milk powder (YCMP) mix during $56 \mathrm{~d}$ of storage at $4 \pm 1^{\circ} \mathrm{C}$.

during the 8-wk storage is shown in Figure 3. The treatment $\times$ day interaction effect $(P=0.99)$ and treatment effect $(P=0.48)$ were not significant, whereas the day effect was significant $(P<0.0001)$. Commercial yogurt powder reconstituted yogurt had detectable yeast and mold counts 1 wk earlier than YCMP reconstituted yogurt, but no detectable counts were obtained in the first 2 wk after the reconstitution (Figure 3). Although one would not be expected to keep the reconstituted yogurt for more than $1 \mathrm{wk}$, this study was extended to determine what would happen if reconstituted products were stored that long. Both the CYP and YCMP reconstituted yogurts were safe in the first week of refrigerated storage.

\section{Apparent Viscosity}

The apparent viscosity of CYP and YCMP mix reconstituted yogurts during the 8 wk of storage is shown in Figure 4 . The treatment $\times$ day interaction effect $(P=0.29)$ and day effect $(P=0.31)$ were not significant, whereas the treatment effect was significant $(P<$ 0.0001).

The mean apparent viscosity of YCMP mix reconstituted yogurt was significantly $(P<0.05)$ higher than the mean apparent viscosity of CYP reconstituted yogurt. It is not likely that the CYP reconstituted yogurt contained stabilizers such as pectin, as the CYP reconstituted yogurt was unstable, forming 2 distinct layers of settled solids and serum on top after $3 \mathrm{~d}$ of reconstitution, and these layers progressed with time. In contrast, YCMP mix reconstituted yogurt was uniform during the entire $56 \mathrm{~d}$ of refrigerated storage, showing no whey separation. Flavored yogurt drinks are made with pectin to improve and stabilize the viscosity (Tamime and Robinson, 1985). Basak and Ramaswamy (1994) reported that pectin and strawberry concentrate had a considerable effect on the flow behavior with yield stress of stirred yogurt.

\section{pH}

The $\mathrm{pH}$ of CYP reconstituted yogurt and YCMP mix reconstituted yogurts during the 8 wk of storage is shown in Figure 5. The treatment $\times$ day interaction effect was not significant $(P=0.64)$, whereas the treatment effect $(P<0.0001)$ and day effect $(P=0.0033)$ were significant.

The mean $\mathrm{pH}$ value of YCMP mix reconstituted yogurt was significantly $(P<0.05)$ higher than the mean $\mathrm{pH}$ value of $\mathrm{CYP}$ reconstituted yogurt. The $\mathrm{pH}$ value observed at d 5 was significantly $(P<0.05)$ higher than the $\mathrm{pH}$ observed at $\mathrm{d} 42,49$, and 56. Gueimonde et al. (2003) reported a decrease in $\mathrm{pH}$ over storage when they studied the quality of plain yogurt stored at $4^{\circ} \mathrm{C}$ for $44 \mathrm{~d}$. A decrease in $\mathrm{pH}$ during yogurt shelf life

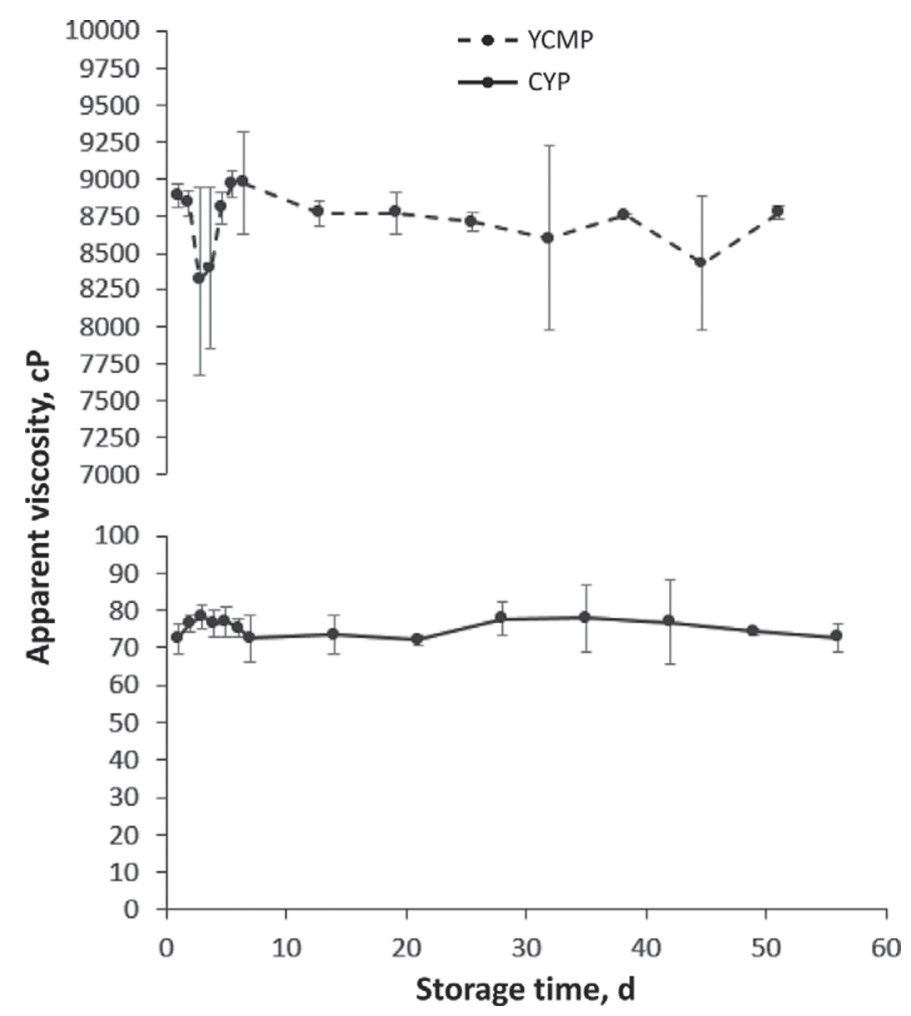

Figure 4. Mean apparent viscosity $\pm \mathrm{SD}$ of reconstituted yogurts from commercial yogurt powder (CYP) and yogurt cultured milk powder (YCMP) mix during $56 \mathrm{~d}$ of storage at $4 \pm 1^{\circ} \mathrm{C}$. 


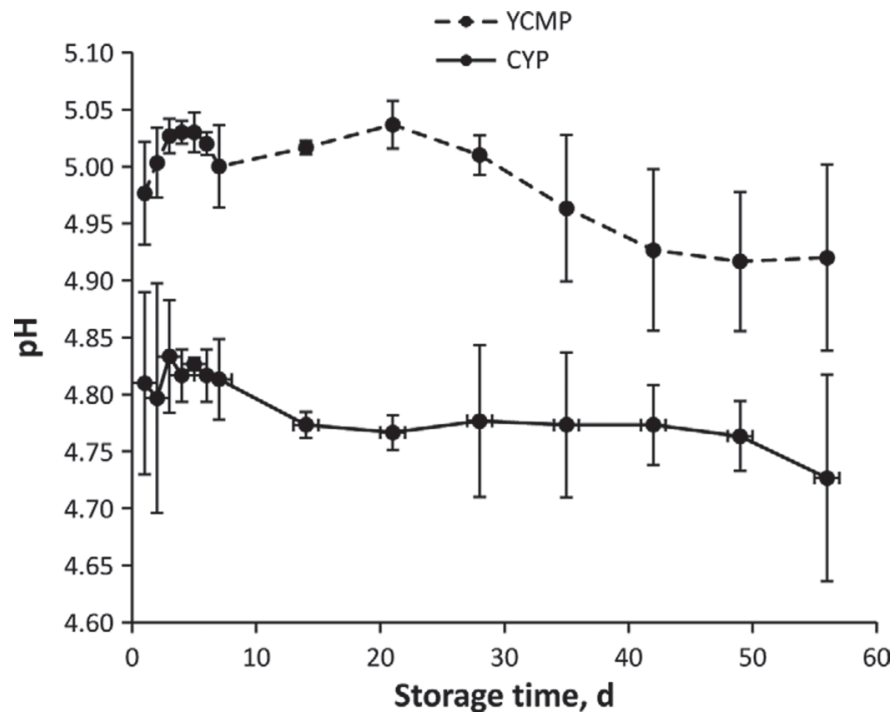

Figure 5. Mean $\mathrm{pH} \pm \mathrm{SD}$ of reconstituted yogurts from commercial yogurt powder (CYP) and yogurt cultured milk powder (YCMP) mix during $56 \mathrm{~d}$ of storage at $4 \pm 1^{\circ} \mathrm{C}$.

is expected as a result of the activity of yogurt starter cultures (Damin et al., 2009).

\section{$T A$}

The TA of CYP and YCMP mix reconstituted yogurts during the 8 wk of storage is shown in Figure 6 . The treatment $\times$ day interaction effect was not significant $(P=0.56)$, whereas the treatment effect $(P=$ $0.0008)$ and day effect $(P<0.0001)$ were significant.

The mean of TA of CYP reconstituted yogurt was significantly $(P<0.05)$ higher than YCMP mix reconstituted yogurt. The TA observed during the first 3 wk were significantly $(P<0.05)$ lower than the TA observed during the last $3 \mathrm{wk}$. The TA increased from 1.23 to 1.29 during 8 wk of refrigerated storage. The TA increase during the storage period follows the decrease in $\mathrm{pH}$. The decrease in $\mathrm{pH}$ during the reconstituted yogurt shelf life resulting from the activity of starter cultures would explain the increase in TA during its shelf life.

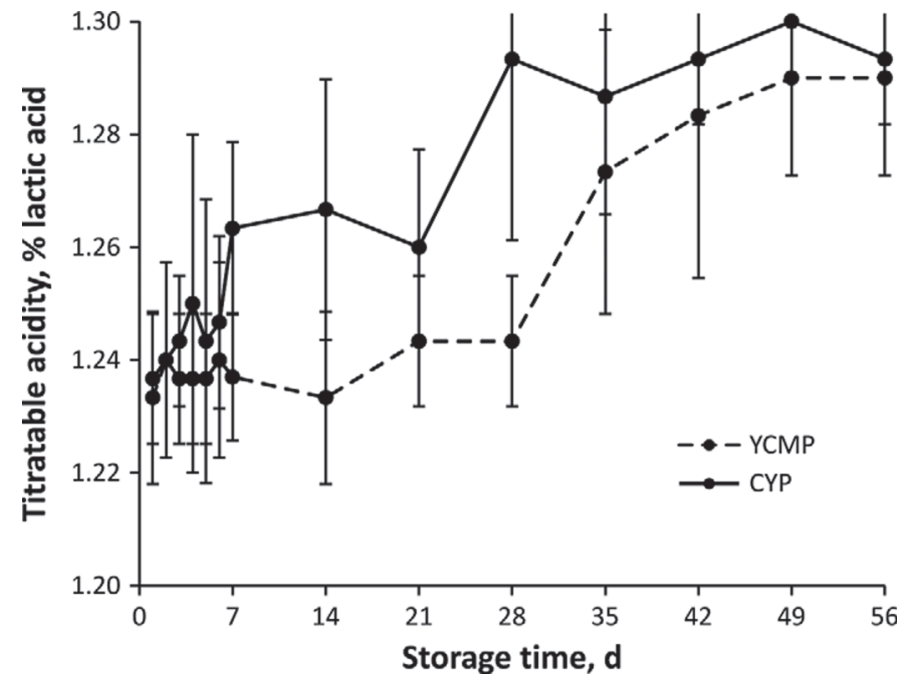

Figure 6. Mean titratable acidity $\pm \mathrm{SD}$ of reconstituted yogurts from commercial yogurt powder (CYP) and yogurt cultured milk powder (YCMP) mix stored for $56 \mathrm{~d}$ at $4^{\circ} \mathrm{C} \pm 1^{\circ} \mathrm{C}$.

\section{Sensory Evaluation}

The sensory evaluation of CYP reconstituted yogurt, YCMP mix reconstituted yogurt, and YCMPA mix reconstituted yogurt was conducted with 100 random people. Probabilities for fixed effect of sensory attributes were appearance $(P<0.0001)$, color $(P=0.0009)$, aroma $(P=0.0025)$, taste $(P<0.0001)$, thickness $(P$ $<0.0001)$, powderiness $(P<0.0001)$, and overall liking $(P<0.0001)$, indicating a significant difference among treatments.

In terms of all attributes (appearance, color, aroma, taste, thickness, powderiness, and overall liking), CYP reconstituted yogurt obtained significantly lower scores compared with the other 2 reconstituted yogurts (Table 2). Compared with YCMP mix reconstituted yogurt, YCMPA mix reconstituted yogurt had significantly higher scores for appearance, taste, powderiness, and overall liking. No significant differences existed in color, aroma, and thickness between YCMP mix reconstituted yogurt and YCMPA mix reconstituted yogurt (Table 2).

Table 2. Means \pm SD for sensory attributes for commercial yogurt powder (CYP), yogurt cultured milk powder (YCMP) mix, and YCMP mix with Lactobacillus acidophilus (YCMPA) reconstituted yogurts ${ }^{1}$

\begin{tabular}{|c|c|c|c|c|c|c|c|}
\hline Treatment & Appearance & Color & Aroma & Taste & Thickness & Powderiness & Overall liking \\
\hline CYP & $4.20 \pm 1.78^{\mathrm{a}}$ & $5.93 \pm 1.50^{\mathrm{a}}$ & $5.55 \pm 1.97^{\mathrm{a}}$ & $3.49 \pm 2.11^{\mathrm{a}}$ & $3.10 \pm 1.74^{\mathrm{a}}$ & $3.45 \pm 1.82^{\mathrm{a}}$ & $3.25 \pm 1.72^{\mathrm{a}}$ \\
\hline YCMP & $5.35 \pm 2.07^{\mathrm{b}}$ & $6.40 \pm 1.46^{\mathrm{b}}$ & $6.12 \pm 1.51^{\mathrm{b}}$ & $4.56 \pm 1.99^{\mathrm{b}}$ & $5.08 \pm 2.07^{\mathrm{b}}$ & $4.08 \pm 1.93^{\mathrm{b}}$ & $4.46 \pm 2.04^{\mathrm{b}}$ \\
\hline YCMPA & $5.93 \pm 1.75^{\mathrm{c}}$ & $6.34 \pm 1.65^{\mathrm{b}}$ & $6.18 \pm 1.85^{\mathrm{b}}$ & $5.23 \pm 2.08^{\mathrm{c}}$ & $5.10 \pm 1.96^{\mathrm{b}}$ & $4.59 \pm 1.96^{\mathrm{c}}$ & $4.95 \pm 2.00^{c}$ \\
\hline
\end{tabular}

${ }^{\mathrm{a}-\mathrm{c}}$ Means within a column with no common superscript differ $(P<0.05)$.

${ }^{1}$ Evaluation was done on a 9 -point rating scale, ranging from $1=$ dislike extremely to $9=$ like extremely. 
According to Routray and Mishra (2011), aroma and taste are the most important sensory characteristics of yogurt. Citric acid is added in the manufacture of commercially available flavored yogurts. Using citric acid in the YCMP mix formulation might have positively influenced the taste of YCMP mix reconstituted yogurt and YCMPA mix reconstituted yogurts over CYP reconstituted yogurt.

The frequency of selecting acceptable was $54 \%$ for the YCMP mix reconstituted yogurt and $59 \%$ for YCMPA mix reconstituted yogurt, more than twice the frequency of $22 \%$ for the CYP reconstituted yogurt. Purchase intent frequencies of CYP, YCMP, and YCMPA reconstituted yogurts before knowing they contained bacteria that provide health benefits were 7,24 , and $24 \%$ respectively. The purchase intent of YCMP mix and YCMPA mix reconstituted yogurts was more than 3 times that of the CYP yogurt. Purchase intent frequency increased from 24 to $42 \%$ for YCMP mix reconstituted yogurt and from 24 to $46 \%$ for YCMPA mix reconstituted yogurts after knowing these reconstituted yogurts contained bacteria that provide health benefits.

\section{CONCLUSIONS}

This study compared the characteristics of YCMP mix reconstituted yogurt with those of CYP reconstituted yogurt. Results of this study showed that YCMP mix reconstituted yogurt had $5 \log \mathrm{cfu} / \mathrm{mL}$ higher counts of $S$. thermophilus compared with the control (CYP reconstituted yogurt) at $56 \mathrm{~d}$. Also, the L. delbrueckii ssp. bulgaricus count of YCMP mix reconstituted yogurt at $28 \mathrm{~d}$ was $6.55 \log \mathrm{cfu} / \mathrm{mL}$ and at $56 \mathrm{~d}$ was $5.35 \log$ $\mathrm{cfu} / \mathrm{mL}$, whereas the CYP reconstituted yogurt at $28 \mathrm{~d}$ onwards had no detectable counts. Commercial yogurt powder reconstituted yogurt did not meet the recommended yogurt culture bacteria concentration of 6 to 8 $\log \mathrm{cfu} / \mathrm{g}$, which was met by YCMP mix reconstituted yogurt. No coliforms were observed at $56 \mathrm{~d}$ for both CYP and YCMP mix reconstituted yogurts. The apparent viscosity of YCMP mix reconstituted yogurt was significantly higher than CYP reconstituted yogurt. Yogurt cultured milk powder mix reconstituted yogurt had significantly higher $\mathrm{pH}$ but significantly lower TA than CYP reconstituted yogurt. Yogurt cultured milk powder mix reconstituted yogurt had significantly higher appearance, color, aroma, taste, thickness, powderiness, and overall liking scores compared with CYP reconstituted yogurt. Consumer acceptability of YCMP mix reconstituted yogurt $(54 \%)$ was more than twice that of CYP reconstituted yogurt $(22 \%)$. Yogurt cultured milk powder mix reconstituted yogurt had markedly higher culture bacterial counts and better physicochemical and sensory characteristics compared with reconstituted commercial yogurt powder.

\section{REFERENCES}

Allen, S. J., B. Okoko, E. Martinez, G. Gregorio, and L. F. Dans. 2009. Probiotics for treating infectious diarrhoea. The Cochrane Collaboration. John Wiley \& Sons Ltd., London, UK.

Ashraf, R., and N. P. Shah. 2011. Selective and differential enumerations of Lactobacillus delbrueckii subsp. bulgaricus, Streptococcus thermophilus, Lactobacillus acidophilus, Lactobacillus casei and Bifidobacterium ssp. in yoghurt-A review. Int. J. Food Microbiol. 149:194-208.

Basak, S., and H. S. Ramaswamy. 1994. Simultaneous evaluation of shear rate and time dependency of stirred yogurt rheology as influenced by added pectin and strawberry concentrate. J. Food Eng. 21:385-393.

Bielecka, M., and A. Majkowska. 2000. Effect of spray drying temperature of yogurt on the survival of starter cultures, moisture content and sensoric properties of yogurt powder. Nahrung 44:257-260.

Boza, Y., D. Barbin, and A. P. R. Scamparini. 2004. Effect of spraydrying on the quality of encapsulated cell of Beijerinckia sp. Process Biochem. 39:1276-1284.

Chandan, R. C. 1999. Enhancing market value of milk by adding cultures. J. Dairy Sci. 82:2245-2256.

Damin, M. R., M. R. Alcântara, A. P. Nunes, and M. N. Oliveira. 2009. Effects of milk supplementation with skim milk powder whey protein concentrate and sodium caseinate on acidification kinetics, rheological properties and structure of nonfat stirred yogurt. Lebensm. Wiss. Technol. 42:1744-1750.

Dave, R. I., and N. P. Shah. 1996. Evaluation of media for selective enumeration of Streptococcus thermophilus, Lactobacillus delbrueckii ssp. bulgaricus, Lactobacillus acidophilus, and bifidobacteria. J. Dairy Sci. 79:1529-1536.

Ebina, T., N. Ogama, and K. Murata. 1995. Antitumor effects of Lactobacillus bulgaricus 878R. Biotherapy 9:65-70.

Gilbreth, S. E., and G. A. Somkuti. 2005. Thermophilin 110: A bacteriocin of Streptococcus thermophilus ST 110. Curr. Microbiol. 51:175-182.

Gilliland, S. E. 1990. Health and nutritional benefits from lactic acid bacteria. FEMS Microbiol. Rev. 7:175-188.

Gueimonde, M., L. Alonso, T. Delgado, J. C. Bada-Gancedo, and C. G. de los Reyes-Gavilán. 2003. Quality of plain yogurt made from refrigerated a $\mathrm{CO}_{2}$-treated milk. Food Res. Int. 36:43-48.

IDFA (International Dairy Foods Association). 2009. Dairy Facts 2009 Edition. IDFA, Washington, DC.

IDFA (International Dairy Foods Association). 2010. Dairy Facts 2010 Edition. IDFA, Washington, DC.

IDFA (International Dairy Foods Association). 2011. Dairy Facts 2011 Edition. IDFA, Washington, DC.

IDFA (International Dairy Foods Association). 2012. Dairy Facts 2012 Edition. IDFA, Washington, DC.

IDFA (International Dairy Foods Association). 2013. Dairy Facts 2013 Edition. IDFA, Washington, DC.

Kabuki, T., H. Uenishi, Y. Seto, T. Yoshioka, and H. Nakajima. 2009. A unique lantibiotic, thermophilin 1277, containing a disulfide bridge and two thioether bridges. J. Appl. Microbiol. 106:853862 .

Kim, S. S., and S. R. Bhowmik. 1990. Survival of lactic acid bacteria during spray drying of plain yogurt. J. Food Sci. 55:10081010,1048 .

Kim, S. S., S. G. Shin, K. S. Chang, S. Y. Kim, B. S. Noh, and S. R. Bhowmik. 1997. Survival of lactic acid bacteria during microwave vacuum-drying of plain yoghurt. Lebensm. Wiss. Technol. 30:573-577.

Kitazawa, H., T. Harata, J. Uemura, T. Saito, T. Kaneko, and T. Itoh. 1998. Phosphate group requirement for mitogenic activation of lymphocytes by an extracellular phosphopolysaccharide from Lactobacillus delbrueckii ssp. bulgaricus. Int. J. Food Microbiol. 40:169-175. 
Kitazawa, H., H. Watanabe, T. Shimosato, Y. Kawai, T. Itoh, and T. Saito. 2003. Immunostimulatory oligonucleotide, CpG-like motif exists in Lactobacillus delbrueckii ssp. bulgaricus NIAI B6. Int. J. Food Microbiol. 85:11-21.

Kumar, P., and H. N. Mishra. 2004. Yoghurt powder-A review of process technology, storage and utilization. Food Bioprod. Process. 82:133-142.

Lee, M. C., L. H. Lin, K. L. Hung, and H. Y. Wu. 2001. Oral bacterial therapy promotes recovery from acute diarrhea in children. Acta Paediatr. Taiwan. 42:301-305.

Michael, M., R. K. Phebus, and K. A. Schmidt. 2010. Impact of a plant extract on the viability of Lactobacillus delbrueckii ssp. bulgaricus and Streptococcus thermophilus in nonfat yogurt. Int. Dairy J. 20:665-672.

Peighambardoust, S. H., A. Golshan Tafti, and J. Hesari. 2011. Application of spray drying for preservation of lactic acid starter cultures: A review. Trends Food Sci. Technol. 22:215-224.

Radaeva, I. A., S. P. Shul'kina, S. I. Kocherga, and B. G. Efron. 1975. Effect of freezing regimes in freeze-drying on yoghurt quality. Molochnaya Promyshlennost 5:22-23.
Ross, R. P., C. Desmond, G. F. Fitzgerald, and C. Stanton. 2005. Overcoming the technological hurdles in the development of probiotic foods. J. Appl. Microbiol. 98:1410-1417.

Routray, W., and H. N. Mishra. 2011. Scientific and technical aspects of yogurt aroma and taste: A review. Compr. Rev. Food Sci. Food Safety 10:208-220.

Rybka, S., and K. Kailasapathy. 1997. Effect of freeze drying and storage on the microbiological and physical properties of $\mathrm{AB}$ yogurt. Milchwissenschaft 52:390-394.

Tamime, A. Y., and R. K. Robinson. 1985. Yogurt: Science and Technology. Pergamon Press, Oxford, UK.

Tharmaraj, N., and N. P. Shah. 2003. Selective enumeration of Lactobacillus delbrueckii ssp. bulgaricus, Streptococcus thermophilus, Lactobacillus acidophilus, bifidobacteria, Lactobacillus casei, Lactobacillus rhamnosus, and propionibacteria. J. Dairy Sci. 86:22882296.

Vasiljevic, T., and N. P. Shah. 2008. Probiotics-From Metchnikoff to bioactives. Int. Dairy J. 18:714-728. 